\section{Does induced masseter muscle pain affect integrated jaw-neck movements similarly in men and women?}

\author{
Wiesinger B, Häggman-Henrikson B, Hellström F, Englund E, Wänman A. Does \\ induced masseter muscle pain affect integrated jaw-neck movements similarly in men \\ and women?
}

Eur J Oral Sci 2016; 124: 546-553. (C) 2016 Eur J Oral Sci

Normal jaw opening-closing involves simultaneous jaw and head-neck movements. We previously showed that, in men, integrated jaw-neck movements during jaw function are altered by induced masseter muscle pain. The aim of this study was to investigate possible sex-related differences in integrated jaw-neck movements following experimental masseter muscle pain. We evaluated head-neck and jaw movements in 22 healthy women and 16 healthy men in a jaw opening-closing task. The participants performed one control trial and one trial with masseter muscle pain induced by injection of hypertonic saline. Jaw and head movements were registered using a three-dimensional optoelectronic recording system. There were no significant sex-related differences in jaw and head movement amplitudes. Head movement amplitudes were significantly greater in the pain trials for both men and women. The proportional involvement of the neck motor system during jaw movements increased in pain trials for 13 of 16 men and for 18 of 22 women. Thus, acute pain may alter integrated jaw-neck movements, although, given the similarities between men and women, this interaction between acute pain and motor behaviour does not explain sex differences in musculoskeletal pain in the jaw and neck regions.

\author{
Birgitta Wiesinger ${ }^{1,2}$, Birgitta \\ Häggman-Henrikson ${ }^{1,3}$, Fredrik \\ Hellström ${ }^{4}$, Erling Englund ${ }^{2}$, \\ Anders Wänman
}

${ }^{1}$ Department of Odontology, Clinical Oral Physiology, Umeå University, Umeå, Sweden; ${ }^{2}$ Department of Research and Development, Umeå University, Sundsvall, Sweden; ${ }^{3}$ Department of Orofacial Pain and Jaw Function, Malmö University, Malmö, Sweden ${ }^{4}$ Centre for Musculoskeletal Research, Department of Occupational and Public Health Sciences, University of Gävle, Umeå, Sweden

Birgitta Wiesinger, Department of Research and Development, Umeå University, Sundsvall Hospital, 85186 Sundsvall, Sweden

E-mail: birgitta.h@wiesinger.se

Key words: head movements; jaw; masseter muscle; neck; pain

Accepted for publication September 2016
Pain and movement are closely linked, and a variety of models have tried to explain changes in motor behaviour associated with pain (1-4). A recent theory on the adaptation of motor control to pain (5) may explain the complexity of changes observed in motor behaviour. This theory suggests a wide spectrum of adaptation mechanisms, including redistribution of activity within and between muscles, as well as changes at different levels of the motor system (5-8). Both taskdependent and individual variability in adaptations have also been observed (9-12). Moreover, sex differences may contribute to individual variability. From both a clinical and an epidemiological perspective, pain affects women significantly more than it affects men $(13,14)$. However, knowledge of the possible differences between men and women in the effects of pain on motor control is still limited.

The effect of experimental muscle pain on human motor control in spinally innervated areas has been extensively studied. The results show that motor activity is redistributed within and between muscles during both single-joint (15) and multisegmental motor tasks, such as walking (16). In a sustained contraction task during experimental pain the pattern of trapezius muscle activation was shown to differ between men and women $(17,18)$. A study in the trigeminally innervated area reported facilitation of jaw-stretch reflex responses in men, but not in women, following injection of glutamate into the masseter muscle (19). No significant sex differences in jaw movement velocity, amplitudes (20), or electromyographic activity of jaw muscles were found during different jaw movement tasks in control trial and in experimentally induced pain (12).

Animal studies have demonstrated a close neurophysiological association between the jaw and neck regions, with trigeminal and cervical afferents converging in the trigeminal brainstem sensory nuclei and upper cervical nociceptive neurons (21-23). A functional relationship between the jaw and neck motor systems has been confirmed in humans (24). Normal jaw function is the result of integrated jaw and head-neck movements during jaw opening-closing tasks (24). The results from a small study sample of healthy subjects suggest significantly smaller head movement amplitudes for women during a continuous maximal jaw opening-closing task (25). We have recently shown that injection of hypertonic saline (HS) in the masseter muscle in men altered the integrated movements by increasing the head-neck movement amplitude in a continuous jaw opening-closing task (26). Many studies report higher sensitivity to HS-induced muscle pain in women than in men (13), even though the results are not consistent (27). Because 
women may perceive experimentally induced pain to be more intensive than such pain is perceived by men (13, 28), a related change in motor strategy may also differ between the sexes.

The aims of this study were to investigate: (i) the effect of experimental masseter muscle pain on integrated jaw-neck movements during jaw openingclosing tasks in women, and (ii) whether there are sex-related differences, by comparing the results with data previously collected from men (26). The hypotheses were: (i) experimental masseter muscle pain in women alters the integrated jaw-neck movements by increased involvement of the neck component during jaw opening-closing, and (ii) the integrated jaw-neck movements during jaw opening-closing tasks differ between men and women.

\section{Material and methods}

\section{Participants}

The study group consisted of 22 women (19-33 yr of age; median age $21 \mathrm{yr}$ ) who were compared with 16 men (20$29 \mathrm{yr}$ of age; median age $22 \mathrm{yr}$ ), as previously reported (26). Exclusion of subjects was based on a screening questionnaire and a clinical examination of the jaw function. Exclusion criteria were:

By questionnaire:

- symptoms in the jaw [tiredness/stiffness, pain, difficulties opening wide, temporomandibular joint (TMJ) sounds, TMJ locking, and impaired opening] once a week or more;

- pain in the head, neck, shoulders, or upper or lower back, once a week or more;

- ear disease, hearing loss, impaired balance, speech problems, diabetes, neurological disorders, muscle and joint disease, or tumours;

- body mass index $\geq 30$;

- elite athletes and persons with very low levels of physical activity;

- pregnancy.

By clinical examination:

- clinical signs and symptoms of temporomandibular disorders (TMD) according to the Research Diagnostic Criteria for TMD, Axis I (29).

Data for men were collected separately and have in part been analysed and previously reported (26). New analyses were also performed to meet the aim of the present paper.

The participants had to abstain from alcohol and analgesics for $24 \mathrm{~h}$ before the experiment. The study was conducted according to the Declaration of Helsinki and was approved by the Regional Ethical Review Board in Umeå. Participants provided written, informed consent.

\section{Experimental procedure}

The participants were given standardized information regarding the procedure before and during the experiments. They were told that the injection was expected to cause pain but were unaware of the measurements of the head-neck movements and the purpose of the study. They were seated in an upright position with firm back support, but without head support, to allow free head-neck movements.

In the trials, the participants performed continuous jaw opening-closing movements from light tooth contact in the intercuspal position to an individual target position and were instructed to keep their eyes closed during the trials. Each participant performed two consecutive control trials without pain induction and two subsequent trials with induced pain in the masseter muscle. The first pain trial started 1 min after pain induction and the second pain trial started 2 min after pain induction. The first control trial was considered a learning trial and the second pain trial was performed at lower pain intensity; therefore, these trials were excluded from the analyses. Hence, only the second control trial (Control) and the first pain trial (Pain) were used in the analyses. The recording time for each trial was $25 \mathrm{~s}$. The experimental procedure is presented in Fig. 1.

The target jaw-opening position was defined as $75 \%$ of individual maximum jaw opening. The maximum jaw opening was measured for each participant and the individual $75 \%$ target position was calculated. Cellular plastic blocks were then cut to fit each individual's jaw opening target (Fig. 1). The main task (i.e. jaw opening to the target position) was practiced with the aid of the plastic block before the first pain trial and first control trial. The participants held the plastic block between their front teeth while the target position was recorded twice using the MacReflex (Qualisys, Gothenburg, Sweden) system, thus documenting the $75 \%$ target position. The participants then practiced the desired pace of the jaw opening-closing cycles with the aid of a metronome set at 50 beats $\min ^{-1}$. The participants were instructed to try to maintain a similar pace during the trials.

\section{Movement recording}

Movements of the lower jaw and the head were recorded simultaneously in three dimensions using a wireless optoelectronic system at a sampling rate of $50 \mathrm{~Hz}$ (MacReflex; Qualisys) (30). Two cameras recorded the movements of a tripod of retro-reflective markers attached to the bridge of the nose (head movements) and a single marker on the chin (lower jaw movements). Details of the set-up have been described previously $(25,26)$.

\section{Pain induction and assessment}

Pain was induced with a unilateral single bolus injection of HS $(0.2 \mathrm{ml}, 5.8 \%)$ into the mid-portion of the masseter muscle with a 27 gauge $\times 3 / 4$ " needle over $15 \mathrm{~s}$. The side of injection was randomized in a balanced order.

Participants were instructed to rate the intensity of the induced pain on a $100 \mathrm{~mm}$ visual analogue scale, marked with the end points 'no pain' $(0)$ and 'worst possible pain imaginable' (100). The pain ratings started $15 \mathrm{~s}$ after the completion of the injection and were repeated every $15 \mathrm{~s}$ before, between, and after the trials, up to $4 \mathrm{~min} 45 \mathrm{~s}$ after the injection.

\section{Lower jaw and head movements}

Jaw movements are the outcome of the combined movements of a lower jaw depression and a head extension. To 


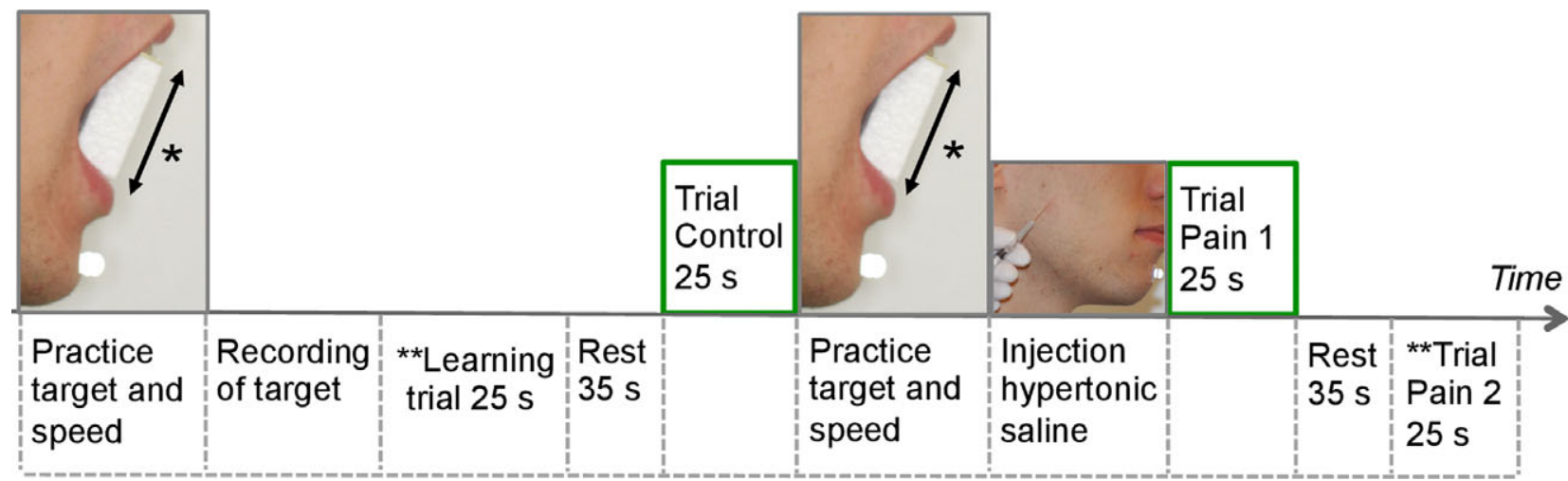

Fig. 1. Schematic overview of the experimental design. *Target jaw opening ( $75 \%$ of maximum jaw opening). **Not used in analysis.

enable mathematical compensation for the associated head-neck movements, reference markers were positioned on the head during the jaw movement recordings. This marker arrangement allowed us to perform a calculation of the lower jaw movements in relation to the head, despite simultaneously occurring head-neck movements. A detailed description has been presented elsewhere (26).

The jaw and head movement amplitudes and cycle times were, for each individual, calculated as an average of the first 10 consecutive cycles in each trial. The following definitions were used (Fig. 2).

- Jaw movement amplitude (in $\mathrm{mm}$ ): the distance from the starting position to the shift from the jaw opening phase to the jaw closing phase.

- Initial head extension (in $\mathrm{mm}$ ): the change in head position at the first jaw movement cycle in relation to the start position.

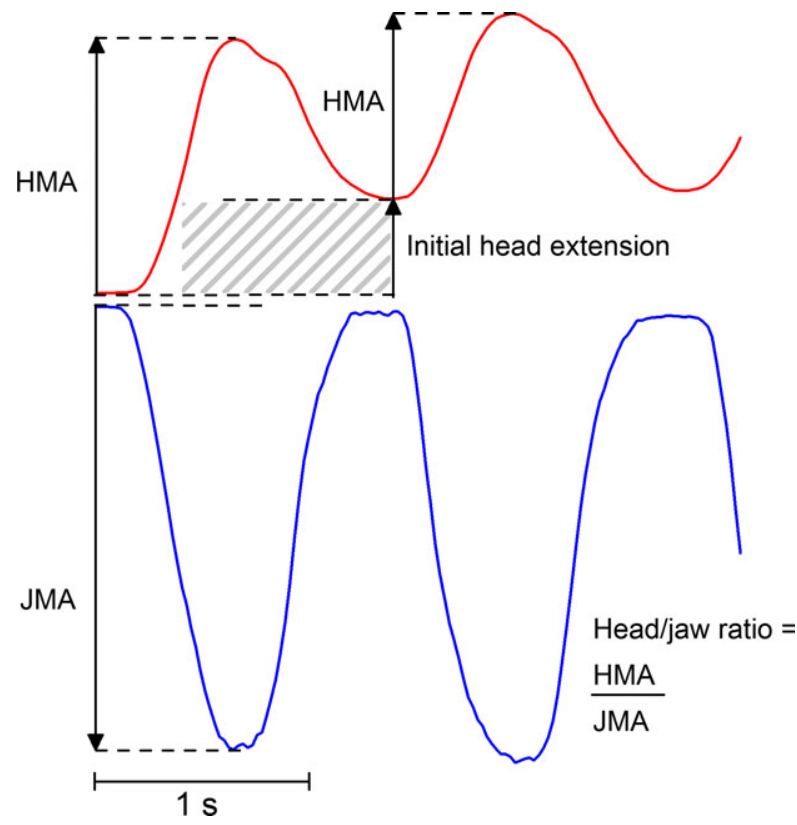

Fig. 2. Schematic definition of jaw movement amplitude (JMA) and head movement amplitude (HMA), initial head extension, and head/jaw ratio, as well as illustration of the first two jaw opening-closing cycles.
- Head movement amplitude (in $\mathrm{mm}$ ): the distance from the starting position to the most superior/posterior position of the head.

- Head/jaw ratio: the proportion between the head movement and the jaw movement amplitudes.

\section{Missing data}

For one man, the jaw and head movement amplitude for one cycle was missing at the end of the recording in the Control trial. This was because of the slower pace of this individual's movement cycles, meaning that during the recording window of $25 \mathrm{~s}$, only nine movement cycles were registered. During the visual inspection of the data, the head movement amplitudes for this subject showed stabilization after the first three cycles. Therefore, in the analysis, the missing value for the 10th cycle was imputed by calculating the mean of the preceding cycles, but excluding cycles $1-3$.

With regard to cycle times, the same man with the missing movement amplitudes also had two missing cycles in the Control trial and one woman had one missing cycle in the Pain trial. These missing data were also a result of slow opening-closing movements, and they could affect the mean cycle time at the group level. No stabilization of cycle times occurred; therefore, the value immediately before the missing data was used to impute the missing value.

\section{Analysis}

Some of the data for the men have been reported previously (26), but in the present paper different analyses were performed. Two-way ANOVA with repeated measures were performed to measure sex-related effects and changes in movement amplitudes for head, jaw, and initial head extension, as well as changes in head/jaw ratio and cycle times. The trial condition (Control and Pain) was entered as a repeated measure and sex was entered as a grouping variable. Data were log-transformed if not normally distributed, as verified by the Shapiro-Wilk normality test.

The jaw movement amplitudes at Control trial and at Pain trial were compared with the individual $75 \%$ target position and tested using the paired samples $t$-test. Pearson correlation was used to analyse the association between accomplishment of the $75 \%$ target position and pain rating $30 \mathrm{~s}$ after the injection of HS. The independent 
samples $t$-test was used to compare the proportional jaw opening amplitudes in relation to the $75 \%$ target position, between men and women, at Control trials and at Pain trials.

Descriptive analysis was used to describe the accuracy of jaw movements (percentage of individual target and SD) and cycle times (geometric mean and 95\% CI, as data were log-normal). The pain intensity ratings for both men and women were compared at all measurement time points after injection of HS, using the Mann-Whitney $U$-test. For all tests used, the level for significance was set at 0.05

\section{Results}

\section{Pain ratings}

All participants reported local pain following injection of HS into the masseter muscle (Fig. 3). There were no statistically significant differences between men and women for the pain intensity rating at any time point at which it was measured after injection.

\section{Jaw and head movement amplitudes}

There were no significant differences between jaw movement amplitudes in Control and Pain trials $[F$ $(1,36)=2.01, P=0.165]$, and no significant sex-related effects $[F(1,36)=0.77, P=0.387]$ (Fig. 4A). The initial head extension (Fig. 4B), as well as head movement amplitudes (Fig. 4C), were significantly larger during Pain trials compared with Control trials $[F(1,36)=$ 17.69, $P<0.001$ and $F(1,36)=23.19, \quad P<0.001$, respectively], with no significant sex-related effects

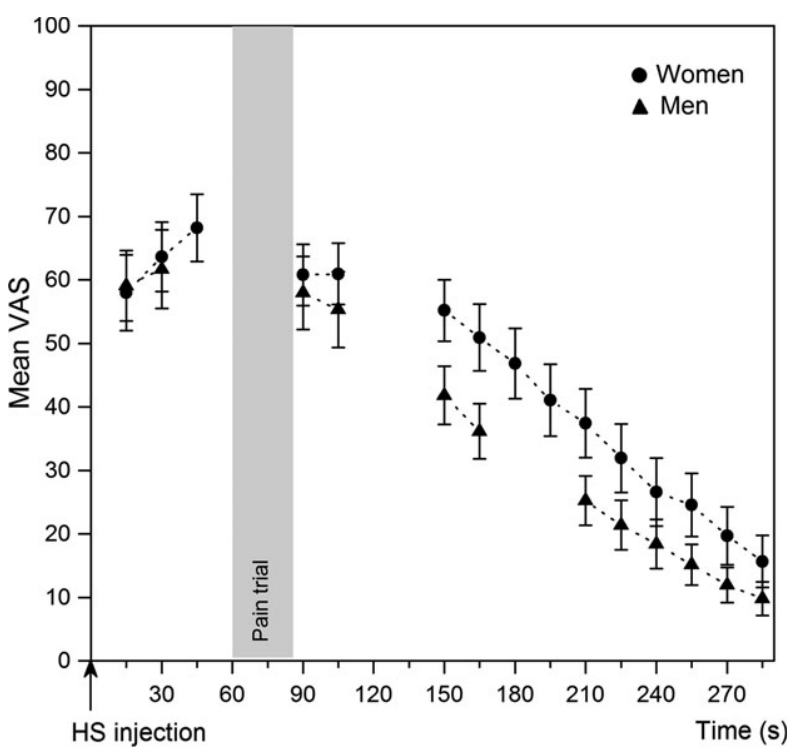

Fig. 3. Pain intensity ratings for men and women. Intensity of pain was rated on a visual analogue scale (VAS) of $0-100 \mathrm{~mm}$ following injection of hypertonic saline (HS) into the masseter muscle. The shaded area indicates the Pain trial, and the $x$-axis shows the period of time after injection. The values are given as mean \pm 1 standard error of pain intensity rating.
$[F(1,36)=0.14, \quad P=0.714 \quad$ and $\quad F(1,36)=0.004$, $P=0.951$, respectively].

The ratio between head and jaw movement amplitudes increased during the Pain trial for 13/16 men and for $18 / 22$ women, and was significantly larger during the Pain trial than during the Control trial $[F$ $(1,36)=19.62, \quad P<0.001] ;$ there were no significant sex-related effects $[F(1,36)=0.3, P=0.589]$ (Fig. 5).

\section{Jaw movement accuracy}

Achievement of the individual $75 \%$ target position did not differ between men and women in any of the trials (Control trial, $P=0.768$; Pain trial, $P=0.216$ ). In the Control trial, men and women showed a significant undershoot of the jaw opening relative to the individual $75 \%$ target position $(P=0.029$ and $P<0.001$, respectively). During the Pain trial, women had a significant undershoot $(P=0.022)$, whereas for men there was no difference between target position and the jaw movement amplitude $(P=0.397)$. Table 1 shows the mean jaw movement accuracy for men and women during Control and Pain trials.

For men there were significant associations between the pain intensity rating $30 \mathrm{~s}$ after injection of $\mathrm{HS}$ and achievement of the individual $75 \%$ target position during the Pain trial $(P=0.006)$ (Fig. 6). This association was not present among the women.

\section{Cycle times}

There was no significant difference between cycle times in the Control and Pain trials $[F(1,36)=0.007$, $P=0.935]$, but the effect for men and women differed significantly $[F(1,36)=4.551, P=0.04]$. The cycle times decreased for men and increased for women during the Pain trial. Table 1 shows the geometric mean and 95\% CI of cycle times for men and women during Control and Pain trials.

\section{Discussion}

This is the first study to investigate possible sex differences in integrated jaw-neck movements from induced masseter muscle pain. The main finding was that men and women employ similar patterns for integrated jaw-neck movements during jaw opening-closing in masseter muscle pain, with increased involvement of the neck component. These results suggest that the immediate jaw-neck motor behaviour response to acute pain is similar in men and women during a goal-orientated jaw task.

Both similarities and differences between men and women in motor strategy have been reported in the trigeminal region. Glutamate injections into the masseter muscle significantly facilitated jaw-stretch reflex responses in men but not in women (19). During HSinduced masseter muscle pain, no differences in electromyographic activity (12) or amplitudes (20) were observed between men and women in opening-closing, protrusion, and contralateral jaw movements. 

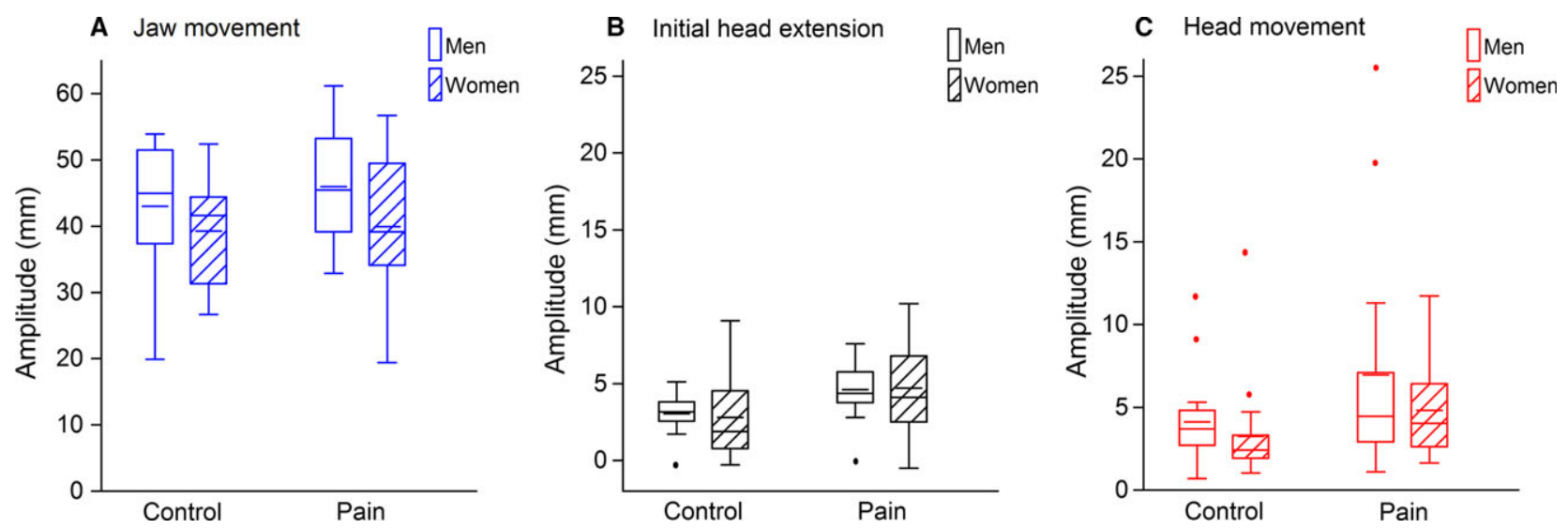

Fig. 4. Box plots for men and women (median, mean, quartiles, minimum, maximum, and outliers) of (A) jaw movement amplitudes, (B) initial head extensions, and (C) head movement amplitudes during the jaw opening-closing task in the Control trial and in the Pain trial.

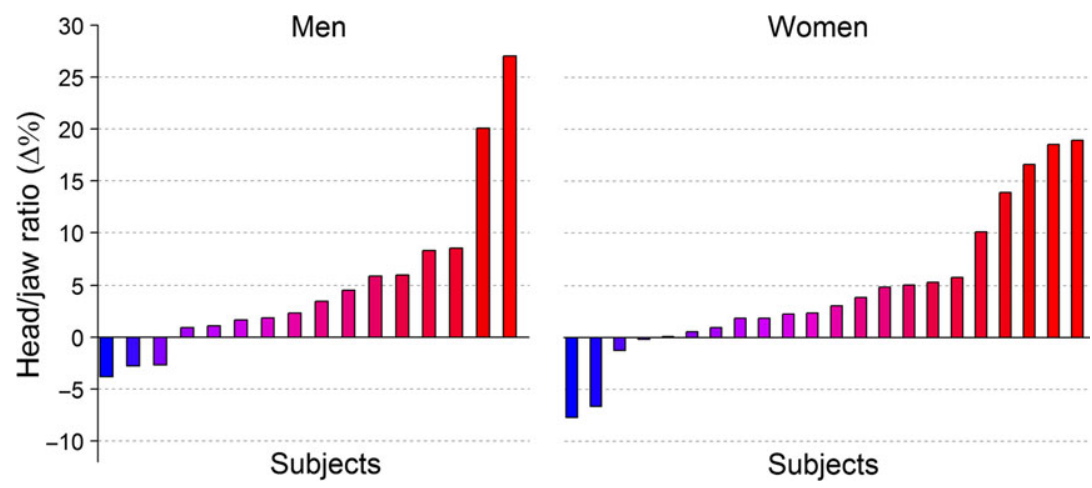

Fig. 5. Difference in head/jaw ratio $(\Delta \%)$ between the Pain trial and the Control trial during the jaw opening-closing task. Bars with positive values represent a higher head/jaw ratio during pain, whereas negative values represent a lower ratio.

Table 1

Jaw movement accuracy and cycle times for men $(\mathrm{n}=16)$ and women $(\mathrm{n}=22)$

\begin{tabular}{lll}
\hline Variable & \multicolumn{1}{c}{ Men } & Women \\
\hline Jaw movement accuracy* & & \\
$\quad$ Control trial & $89.8(17.4)$ & $88.3(12.5)$ \\
$\quad$ Pain trial & $96.8(16.1)$ & $89.5(19.4)$ \\
Cycle time & & \\
$\quad$ Control trial & $1.50(1.30-1.73)$ & $1.48(1.36-1.61)$ \\
Pain trial & $1.40(1.30-1.52)$ & $1.57(1.42-1.73)$
\end{tabular}

*Jaw movement accuracy as a percentage of the individual target. Values are given as mean (SD).

${ }^{\dagger}$ Cycle times for men and women during Control and Pain trials. Values are given as geometric mean $(95 \% \mathrm{CI})$.

For the neck region, men and women showed different patterns of trapezius muscle activation during a sustained contraction during experimental trapezius muscle pain $(17,18)$. At a glance, these findings seem to contradict the results of the present study when investigating jaw or neck motor systems separately. However, normal jaw function is the result of integrated jaw and head-neck movements during jaw opening-closing tasks
(24) and it is unlikely that the central nervous system (CNS) controls individual muscles (31), but rather uses motor modules, like muscle synergies (32).

The aim of the present study was not to analyse isolated movements in the jaw-neck motor system, but instead to study the final outcome of the combined jaw-head movements at a task level. Indeed, if one part of a multi-joint system is fixed, the remaining parts will be affected. This has been demonstrated specifically for the jaw-neck motor system, in which fixation of the head resulted in a reduction of lower jaw movement amplitudes (33). Individual muscles, joint torques, and joint angles cannot be controlled independently but require to be controlled together in a task-specific way (34). A strong task dependency in the trigeminal system has been supported by the proportional involvement of the neck in jaw motor tasks in relation to both task $(25,33)$ and peripheral input $(35)$. Rhythmic jaw movements (e.g. chewing) are governed by a central pattern generator which provides a basic temporal and spatial pattern that is modified by peripheral feedback (36). Also, in other movements characterized by a significant influence of central pattern generators, such as pedalling and walking, the roles of muscle synergies allow 

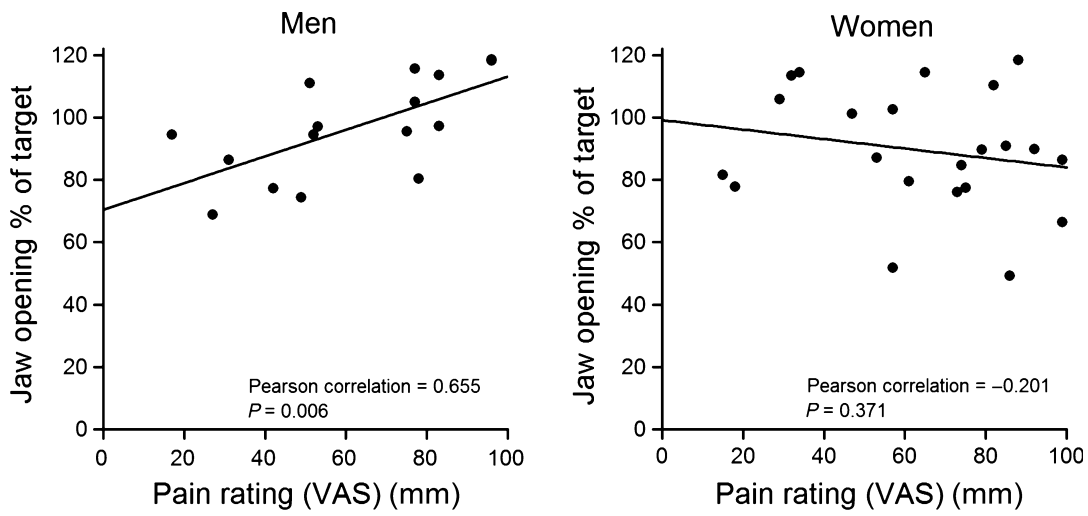

Fig. 6. Association between pain intensity rating after injection of hypertonic saline (HS) and achievement of the target position during the Pain trial, calculated using Pearson correlation. The $y$-axis represents the proportional jaw opening amplitude in relation to the individual target position during the jaw opening-closing task in induced pain. The individual target position is represented by $100 \%$ on the $y$-axis. The $x$-axis represents the rating of pain intensity $30 \mathrm{~s}$ after injection of HS, measured on a visual analogue scale (VAS) of 0-100 mm. Each dot represents one individual. There was a positive, significant correlation between pain rating and accomplishment of target position for men.

for robust motor behaviours across movement parameters, such as speed $(37,38)$. In other multi-joint systems, such as those involved in balance control, this is also evident because muscle synergies are consistent across biomechanical contexts (39). It should be mentioned that modular control of movement through muscle synergies has not been investigated in the jaw-neck system, and the number of possible muscle synergies are unknown. There are a substantial number of muscles that could participate in achieving tasks related to positioning the gape, and subtle differences in integrated jaw-neck movements may emerge from altering muscle activation patterns.

The present study showed that experimental muscle pain affected movements in the jaw-neck motor system similarly in men and women. Experimental muscle pain in limbs shows that muscle synergies may be shared between painful and non-painful conditions, and may also be condition-specific (40). One important aspect of how the CNS controls muscle synergies is the role of afferent information, which has been suggested to adapt the recruitment of muscle synergies (41). Studies in animals show that activation of nociceptors in the masseter muscle changes not only proprioceptive signals from the masseter itself (42) but also input from the trapezius muscle (43), which thus provides a foundation for adopting movement strategies to achieve task objectives.

In contrast to previous studies, which indicated reduced jaw movement amplitudes following experimental pain during jaw opening (20) and chewing (44), the present study showed no significant changes in jaw movement amplitudes for either men or women. This may be related to the fact that our study had a specific goal-orientated jaw-opening task, which may reduce the influence of the nociceptive input on the final movement outcome. It should be remembered, however, that all of these studies relate to short-term experimental pain and therefore conclusions regarding long-term pain conditions cannot be drawn.
Discrepancies also exist between studies into sex differences in the perception of pain intensity. Although most studies report higher pain intensities among women $(17,28,45)$, some studies have found no sexrelated differences in experimental muscle pain (46-48) The mechanisms underlying sex differences in pain responses are not entirely understood but are mostly explained from a biological or psychosocial perspective (13). In the present study, the reported pain intensities did not differ between men and women. However, the results indicate that, for men only, a higher pain rating was associated with larger jaw movement amplitudes in relation to target. Jaw movement amplitudes in relation to target, also referred to as jaw movement accuracy, were, with the exception of men during the pain trial, lower than target, thus undershooting target amplitudes. Undershooting target positions has been shown to be a common motor strategy during limb movements (49) as correcting an undershoot requires less energy than correcting an overshoot (50). However, data from both men and women showed substantial variability of both under- and overshoot. This variability could reflect individual motor control strategies that is either an accuracy-prone or a speed-prone strategy $(51,52)$. A larger study population and a predefined pain cut-off would be necessary for drawing any further conclusions regarding this matter.

The striking similarities between men and women in integrated jaw-neck function during experimental pain reinforce the notion that an integrated jaw-neck function has been preserved during evolution. The importance of the sensorimotor trigeminal-cervical coupling is demonstrated early in the newborn baby, whose feeding is facilitated by the rooting reflex. In a previous study (53), we observed that proprioceptive manipulation of the trigeminal fusimotor system, with the aid of vibration, did not affect jaw movement accuracy during continuous opening-closing. Taken together, these results indicate a high capacity of the jaw-neck motor 
system to achieve a specific position of the gape, regardless of sensory manipulation or distraction. Cognitive processes may thus be involved to suppress or inhibit the reflex response at the motor nucleus level when a specific goal or target is in focus.

\section{Study limitations}

The participants were included or excluded following participation in a screening procedure with a clinical examination and a questionnaire. The research diagnostic criteria for TMD were used to confirm the absence of clinical signs and symptoms of TMD. For accurate diagnosis according to these criteria, imaging has been recommended $(54,55)$ but this was not included in our screening procedure. Subjects with pain in the jaw, head, neck, shoulder, or back were excluded. At the time of the experiment, the absence of pain was confirmed orally, but no written initial pain score was registered. We also excluded participants with a sedentary lifestyle, as well as elite athletes, to avoid extremely divergent motor skills.

Injection of HS is a well-known and widely used method to induce muscular pain. There are no side effects and the pain subsides within minutes (56). However, the perception of pain is not only driven by noxious input. Also, cognitive factors, such as pain expectation, may modulate pain perception (57). DuBÉ et al. (58) found that expectations of pain did not elicit motor inhibition, but acute pain inhibited corticospinal excitability. Thus, we cannot rule out that the participants' expectation of pain influenced the head-neck movements.

Overall, the present study has shown that there are no significant differences between men and women in integrated jaw-neck movements when they try to achieve a specific precision jaw-opening task during experimentally induced masseter muscle pain. This indicates similar alterations in the integrated jaw-neck motor strategy for men and women during acute pain. Owing to the similarities between the results of men and women, interactions between acute pain and motor behaviour do not explain the sex differences observed in clinical pain conditions in the jaw and neck regions.

Acknowledgements - This study was supported by the Department of Research and Development, Västernorrland County Council; The Swedish Dental Society; and The Sigurd and Elsa Golje Memorial Foundation.

Conflicts of interest - No conflicts of interest are declared by the authors.

\section{References}

1. JohANSSON H, SoJKA P. Pathophysiological mechanisms involved in genesis and spread of muscular tension in occupational muscle pain and in chronic musculoskeletal pain syndromes: a hypothesis. Med Hypotheses 1991; 35: 196203.

2. Lund JP, Donga R, Widmer CG, Stohler CS. The painadaptation model: a discussion of the relationship between chronic musculoskeletal pain and motor activity. Can J Physiol Pharmacol 1991; 69: 683-694.

3. Stohler CS. Craniofacial pain and motor function: pathogenesis, clinical correlates, and implications. Crit Rev Oral Biol Med 1999; 10: 504-518.

4. Murray GM, Peck CC. Orofacial pain and jaw muscle activity: a new model. J Orofac Pain 2007; 21: 263-278; discussion 279-288.

5. Hodges PW, Tucker K. Moving differently in pain: a new theory to explain the adaptation to pain. Pain 2011; 152: S90-S98.

6. Hodges PW. Pain and motor control: from the laboratory to rehabilitation. J Electromyogr Kinesiol 2011; 21: 220-228.

7. Hodges PW, Smeets RJ. Interaction between pain, movement, and physical activity: short-term benefits, long-term consequences, and targets for treatment. Clin J Pain 2015; 31: 97-107.

8. Hug F, Hodges PW, van den Hoorn W, Tucker K. Between-muscle differences in the adaptation to experimental pain. J Appl Physiol (1985) 2014; 117: 1132-1140.

9. Hodges PW, Coppieters MW, Macdonald D, Cholewicki J. New insight into motor adaptation to pain revealed by a combination of modelling and empirical approaches. Eur $J$ Pain 2013; 17: 1138-1146.

10. Falla D, Gizzi L, Tschapek M, Erlenwein J, Petzke F. Reduced task-induced variations in the distribution of activity across back muscle regions in individuals with low back pain. Pain 2014; 155: 944-953.

11. Hug F, Hodges PW, Tucker K. Task dependency of motor adaptations to an acute noxious stimulation. J Neurophysiol 2014; 111: 2298-2306.

12. Sae-Lee D, Whittle T, Forte AR, Peck CC, Byth K, Sessle BJ, Murray GM. Effects of experimental pain on jaw muscle activity during goal-directed jaw movements in humans. Exp Brain Res 2008; 189: 451-462.

13. Fillingim RB, King CD, Ribeiro-Dasilva MC, Rahim-Williams B, Riley JL 3rd. Sex, gender, and pain: a review of recent clinical and experimental findings. J Pain 2009; 10: $447-485$.

14. Dao TT, LeResche L. Gender differences in pain. J Orofac Pain 2000; 14: 169-184; discussion 184-195.

15. Ervilha UF, Farina D, Arendt-Nielsen L, Graven-Nielsen T. Experimental muscle pain changes motor control strategies in dynamic contractions. Exp Brain Res 2005; 164: 215-224.

16. van den Hoorn W, Hodges PW, van Dieen JH, Hug F. Effect of acute noxious stimulation to the leg or back on muscle synergies during walking. $J$ Neurophysiol 2015; 113: 244 254.

17. Falla D, Arendt-Nielsen L, Farina D. Gender-specific adaptations of upper trapezius muscle activity to acute nociceptive stimulation. Pain 2008; 138: 217-225.

18. Ge Hy, Arendt-Nielsen L, Farina D, Madeleine P. Gender-specific differences in electromyographic changes and perceived pain induced by experimental muscle pain during sustained contractions of the upper trapezius muscle. Muscle Nerve 2005; 32: 726-733.

19. Cairns Be, Wang K, Hu JW, Sessle BJ, Arendt-Nielsen L, Svensson P. The effect of glutamate-evoked masseter muscle pain on the human jaw-stretch reflex differs in men and women. J Orofac Pain 2003; 17: 317-325.

20. Sae-Lee D, Whittle T, Peck CC, Forte AR, Klineberg iJ, Murray GM. Experimental jaw-muscle pain has a differential effect on different jaw movement tasks. J Orofac Pain 2008; 22: $15-29$.

21. Sessle BJ, Hu JW, Amano N, Zhong G. Convergence of cutaneous, tooth pulp, visceral, neck and muscle afferents onto nociceptive and non-nociceptive neurones in trigeminal subnucleus caudalis (medullary dorsal horn) and its implications for referred pain. Pain 1986; 27: 219-235.

22. Hu JW, Sun KQ, Vernon H, Sessle BJ. Craniofacial inputs to upper cervical dorsal horn: implications for somatosensory information processing. Brain Res 2005; 1044: 93-106.

23. Morch CD, Hu JW, Arendt-Nielsen L, Sessle BJ. Convergence of cutaneous, musculoskeletal, dural and visceral 
afferents onto nociceptive neurons in the first cervical dorsal horn. Eur J Neurosci 2007; 26: 142-154.

24. ERIKSSON PO, Zafar H, Nordh E. Concomitant mandibular and head-neck movements during jaw opening-closing in man. J Oral Rehabil 1998; 25: 859-870.

25. Eriksson PO, Häggman-Henrikson B, Nordh E, Zafar H. Co-ordinated mandibular and head-neck movements during rhythmic jaw activities in man. $J$ Dent Res 2000; 79: 1378-1384.

26. Wiesinger B, Häggman-Henrikson B, Hellström F, Wän MAN A. Experimental masseter muscle pain alters jaw-neck motor strategy. Eur J Pain 2013; 17: 995-1004.

27. Racine M, Tousignant-Laflamme Y, Kloda LA, Dion D, Dupuis G, Choiniere M. A systematic literature review of 10 years of research on sex/gender and experimental pain perception - part 1: are there really differences between women and men? Pain 2012; 153: 602-618.

28. Cairns Be, Hu JW, Arendt-Nielsen L, Sessle BJ, Svensson P. Sex-related differences in human pain and rat afferent discharge evoked by injection of glutamate into the masseter muscle. J Neurophysiol 2001; 86: 782-791.

29. Dworkin SF, LeResche L. Research diagnostic criteria for temporomandibular disorders: review, criteria, examinations and specifications, critique. J Craniomandib Disord 1992; 6: 301-355.

30. Josefsson T, Nordh E, Eriksson PO. A flexible high-precision video system for digital recording of motor acts through lightweight reflex markers. Comput Methods Programs Biomed 1996; 49: 119-129.

31. Bernstein N. The co-ordination and regulation of movements. Oxford: Pergamon Press, 1967.

32. Tresch MC, Saltiel P, Bizzi E. The construction of movement by the spinal cord. Nat Neurosci 1999; 2: 162-167.

33. Häggman-Henrikson B, Nordh E, Zafar H, Eriksson PO Head Immobilization can Impair Jaw Function. $J$ Dent Res 2006; 85: 1001-1005.

34. Scholz JP, Schöner G. The uncontrolled manifold concept: identifying control variables for a functional task. Exp Brain Res 1999; 126: 289-306.

35. Häggman-Henrikson B, Österlund C, Eriksson PO. Endurance during chewing in whiplash-associated disorders and TMD. J Dent Res 2004; 83: 946-950.

36. Lund JP. Mastication and its control by the brain stem. Crit Rev Oral Biol Med 1991; 2: 33-64.

37. Clark DJ, Ting LH, Zajac Fe, Neptune RR, Kautz SA. Merging of healthy motor modules predicts reduced locomotor performance and muscle coordination complexity poststroke. J Neurophysiol 2010; 103: 844-857.

38. McGowan CP, Neptune RR, Clark DJ, Kautz SA. Modular control of human walking: adaptations to altered mechanical demands. J Biomech 2010; 43: 412-419.

39. Torres-Oviedo G, Ting LH. Subject-specific muscle synergies in human balance control are consistent across different biomechanical contexts. $J$ Neurophysiol 2010; 103: 3084-3098.

40. Muceli S, Falla D, Farina D. Reorganization of muscle synergies during multidirectional reaching in the horizontal plane with experimental muscle pain. J Neurophysiol 2014 111: $1615-1630$.

41. Cheung VC, D’Avella A, Tresch MC, Bizzi E. Central and sensory contributions to the activation and organization of muscle synergies during natural motor behaviors. J Neurosci 2005; 25: 6419-6434.

42. Ro JY, CAPRA NF. Physiological evidence for caudal brainstem projections of jaw muscle spindle afferents. Exp Brain Res 1999; 128: 425-434.

43. Hellström F, Thunberg J, Bergenheim M, Suölander P, Pedersen J, Johansson H. Elevated intramuscular concentration of bradykinin in jaw muscle increases the fusimotor drive to neck muscles in the cat. J Dent Res 2000; 79: 1815-1822.
44. Svensson P, Arendt-Nielsen L, Houe L. Sensory-motor interactions of human experimental unilateral jaw muscle pain: a quantitative analysis. Pain 1996; 64: 241-249.

45. Svensson P, Cairns Be, Wang K, Hu JW, Graven-Nielsen T, Arendt-Nielsen L, Sessle BJ. Glutamate-evoked pain and mechanical allodynia in the human masseter muscle. Pain 2003; 101: 221-227.

46. Ge Hy, Madeleine P, Cairns BE, Arendt-Nielsen L. Hypoalgesia in the referred pain areas after bilateral injections of hypertonic saline into the trapezius muscles of men and women: a potential experimental model of gender-specific differences. Clin J Pain 2006; 22: 37-44.

47. Nie H, Arendt-Nielsen L, Kawczynski A, Madeleine P. Gender effects on trapezius surface EMG during delayed onset muscle soreness due to eccentric shoulder exercise. $J$ Electromyogr Kinesiol 2007; 17: 401-409.

48. Torisu T, Wang K, Svensson P, De laat A, Fujil H, Arendt-Nielsen L. Effects of muscle fatigue induced by lowlevel clenching on experimental muscle pain and resting jaw muscle activity: gender differences. Exp Brain Res 2006; 174: 566-574.

49. Elliott D, Dutoy C, Andrew M, Burkitt JJ, Grierson Le, Lyons JL, Hayes SJ, Bennett SJ. The influence of visual feedback and prior knowledge about feedback on vertical aiming strategies. $J$ Mot Behav 2014; 46: 433-443.

50. Elliott D, Hansen S, Grierson Le, Lyons J, Bennett SJ, HAYes SJ. Goal-directed aiming: two components but multiple processes. Psychol Bull 2010; 136: 1023-1044.

51. Adam JJ. The effects of objectives and constraints on motor control strategy in reciprocal aiming movements. $J$ Mot Behav 1992; 24: 173-185.

52. de Grosbois J, Heath M, Tremblay L. Augmented feedback influences upper limb reaching movement times but does not explain violations of Fitts' Law. Front Psychol 2015; 6: 800.

53. Wiesinger B, Häggman-Henrikson B, Wänman A, Lind KVIST M, Hellström F. Jaw-opening accuracy is not affected by masseter muscle vibration in healthy men. Exp Brain Res 2014; 232: 3501-3508.

54. Ahmad M, Hollender L, Anderson Q, Kartha K, Оhrbach R, Truelove EL, John MT, Schiffman EL. Research diagnostic criteria for temporomandibular disorders (RDC) TMD): development of image analysis criteria and examiner reliability for image analysis. Oral Surg Oral Med Oral Pathol Oral Radiol Endod 2009; 107: 844-860.

55. Schiffman E, Ohrbach R, Truelove E, Look J, Anderson G, Goulet JP, List T, Svensson P, Gonzalez Y, Lobbezoo F, Michelotti A, Brooks SL, Ceusters W, Drangsholt M, Ettlin D, Gaul C, Goldberg LJ, Haythornthwaite JA, Hollender L, Jensen R, John MT, De LA, de Leeuw R, Maixner W, van der Meulen M, Murray GM, Nixdorf DR, Palla S, Petersson A, Pionchon P, Smith B, Visscher CM, Zakrzewska J, Dworkin SF, International Rdc/Tmd Consortium Network IafDR, Orofacial Pain Special Interest Group IAftSoP. Diagnostic Criteria for Temporomandibular Disorders (DC/TMD) for clinical and research applications: recommendations of the International RDC/ TMD Consortium Network* and Orofacial Pain Special Interest Groupdagger. J Oral Facial Pain Headache 2014; 28 : 6-27.

56. Graven-Nielsen T, Arendt-Nielsen L, Jensen T. Experimental muscle pain: a quantitative study of local and referred pain in humans following injection of hypertonic saline. J Musculoskelet Pain 1997; 5: 49-69.

57. Atlas LY, Wager TD. How expectations shape pain. Neurosci Lett 2012; 520: 140-148.

58. Dube JA, Mercier C. Effect of pain and pain expectation on primary motor cortex excitability. Clin Neurophysiol 2011; 122: 2318-2323. 